\section{ORIGINAL RESEARCH}

\author{
S.L. Harder \\ K.M. Hopp \\ H. Ward \\ H. Neglio \\ J. Gitlin \\ D. Kido
}

\title{
Mineralization of the Deep Gray Matter with Age: A Retrospective Review with Susceptibility- Weighted MR Imaging
}

\begin{abstract}
BACKGROUND AND PURPOSE: Susceptibility-weighted imaging (SWI) is an advanced MR imaging sequence that can be implemented at high resolution. This sequence can be performed on conventional MR imaging scanners and is very sensitive to mineralization. The purpose of this study was to establish the course of mineralization in the deep gray matter with age by using SWI.
\end{abstract}

\begin{abstract}
MATERIALS AND METHODS: We retrospectively reviewed susceptibility-weighted images of 134 patients (age range, 1 to 88 years). Inclusion criteria comprised a normal conventional MR imaging (T1, $\mathrm{T} 2$, and fluid-attenuated inversion recovery sequences). We statistically analyzed the relative signal intensities of the globus pallidus, putamen, substantia nigra, caudate nucleus, red nucleus, and thalamus for correlation with age. The putamen was graded according to a modified scale, based on previous work that described a systematic pattern of mineralization with age. Bands of hypointensity in the globus pallidus, dubbed "waves," were also evaluated.
\end{abstract}

\begin{abstract}
RESULTS: We documented decreasing intensity (ie, increasing mineralization) with age in all deep gray matter areas analyzed. We confirmed the age-related posterolateral to anteromedial progression of mineralization in the putamen. Characteristic medial and lateral bands of mineralization were exhibited in the globus pallidus in all children and young adults older than 3 years. Finally, an increase in the number of "waves" present in the globus pallidus was associated with increased age by category.

CONCLUSION: This study documents the course and pattern of mineralization in the deep gray matter with age, as determined by SWI. These findings may play a role in evaluating diseased brains in the future.
\end{abstract}

$I^{n}$ 1958, Hallgren and Sourander ${ }^{1}$ performed some of the earliest work on characterizing brain iron. They studied iron distribution in various tissues, including the deep gray matter, during an autopsy of brains from 98 subjects, excluding those with cerebrovascular or neuropsychiatric disorders. Iron was found to increase with age in most brain tissues. In the globus pallidus, red nucleus, substantia nigra, and dentate nucleus, iron increases rapidly from birth until the end of the second decade, plateaus for several years, and then shows another milder increase after age 60. Iron increases more slowly in the putamen and caudate, levelling off in the fifth or sixth decade. Subsequent iron-staining studies have largely supported the work of Hallgren and Sourander. ${ }^{1}$

Most brain iron, other than that found in hemoglobin, is protein-bound, nonheme iron. Approximately one third of nonheme iron is postulated to be in the form of ferritin, though this fraction may be higher in certain deep gray matter structures. Other forms include transferrin, lactoferrin, hemosiderin (thought to be degenerated ferritin), ionic iron, and possibly biogenic magnetite. ${ }^{2,3}$ The 2 most important compounds in brain-iron regulation are transferrin, which is used in iron transport, and ferritin, which is used in iron storage. Transferrin and ferritin are also thought to be the only forms of nonheme iron that have a high enough concentration in the

Received April 5, 2007; accepted after revision June 17.

From the Department of Medical Imaging, Royal University Hospital, Saskatoon, Saskatchewan, Canada.

This study was funded by the Canadian Heads of Academic Radiology (CHAR) and the Department of Medical Imaging, University of Saskatchewan.

Please address correspondence to Sheri Harder, MD, FRCPC, Department of Medical Imaging, Royal University Hospital, 103 Hospital Dr, Saskatoon, SK, Canada, S7N OW8; e-mail: sheri.harder@saskatoonhealthregion.ca

DOI 10.3174/ajnr.A0770 brain to be detected currently by MR. ${ }^{3}$ The mechanisms by which iron and other minerals are deposited in the brain are not well understood. Although the bulk of iron required for the metabolic activity of the adult brain is taken up during the neonatal period, experiments with radioactively labeled iron indicate that small amounts continue to be transported into the adult brain. ${ }^{4}$ In particular, the basal ganglia may exhibit increased susceptibility to mineralization because of their high metabolic rate, and the pattern of mineralization may, in part, relate to the functional vascular components of the striatum. ${ }^{5,6}$ Mineral deposits may, in turn, restrict blood flow and cause neural tissue injury that leads to further mineralization.

It is believed that the destruction of gray matter causes the release of iron, which is then taken up by activated microglia. Studies have linked increased brain mineralization with several diseases (eg, Parkinson, Alzheimer, Huntington, dementia with Lewy bodies, multiple sclerosis, hemochromatosis, Hallervorden-Spatz, Down syndrome, and AIDS). Histochemical analysis of mineralization of the basal ganglia has shown that many other minerals may be present in addition to iron (eg, calcium, manganese, zinc, copper, magnesium, aluminum, potassium, phosphorus). ${ }^{5,7}$ It has been found that the accumulation of iron tends to precede the deposition of calcium and other minerals. These discoveries have underlined the need to develop in vivo imaging techniques sensitive to mineralization, particularly iron.

Early attempts at developing such techniques have involved CT imaging. ${ }^{89}$ More recently, with MR imaging, T2 shortening in the gray matter nuclei with age has been well documented. ${ }^{10-14}$ A reduction in T2 is thought to be predominantly related to iron deposition, particularly ferritin, ${ }^{2,11,15}$ though 1 team ${ }^{16}$ reports contrary findings. The drawback of 
simply using T2 shortening as a measure of mineralization is that it is also affected by factors such as myelin loss and changes in water concentration, which vary with tissue type, presence of disease, and age. ${ }^{3,11,13,17}$ Some researchers have worked on minimizing this effect. Bartzokis et $\mathrm{al}^{11}$ used an imaging process called field-dependent relaxation rate increase (FDRI) to extract that portion of T2 shortening that is the result of mineralization. However, this method is logistically difficult because it requires access to $2 \mathrm{MR}$ machines of different magnetic field strengths as well as careful positioning of the patient in order to extract similar sections from highand low-field sequences for comparison. Gelman et $\mathrm{al}^{13}$ also tried to measure susceptibility using gradient-echo sampling of free induction decay and echo (GESFIDE) MR imaging ${ }^{18}$ to measure $\mathrm{R} 2^{\prime}$, ie, that part of the transverse relaxation rate resulting from magnetic field inhomogeneities $\left(\mathrm{R} 2^{\prime}=\mathrm{R} 2^{\star}-\right.$ $\mathrm{R} 2$, where $\mathrm{R} 2{ }^{*}$ refers to the actual observed relaxation rate, and $\mathrm{R} 2$ refers to the relaxation rate intrinsic to the tissue). They found a correlation between $\mathrm{R} 2$ ' and iron concentration in the brain. However, they experienced problems with significant magnetic field distortions in the region of the sphenoid sinus and nasal cavity.

\section{Susceptibility-Weighted Imaging (SWI)}

Susceptibility-weighted imaging (SWI) is an advanced MR imaging sequence that can be implemented at high resolution. Reichenbach and colleagues ${ }^{19}$ designed this heavily $\mathrm{T}^{*}$ weighted, 3D fast low-angle shot (FLASH) MR imaging technique that can be performed on conventional MR imaging scanners with an imaging time comparable with, or lower than, that of other advanced mineralization sequences. SWI makes use of R2' and phase information because these have been shown to have a stronger and more accurate correlation with brain iron than R2 alone., 13,19-21 SWI was initially designed for MR venography ${ }^{19,22,23}$ with use of the paramagnetic property of deoxyhemoglobin that is also the basis of blood oxygen level-dependent (BOLD) imaging. SWI is very sensitive to mineralization, ${ }^{24}$ with studies showing this sequence to be 4 times more sensitive than a conventional gradient recalled-echo (GRE) sequence in the detection of hemorrhage. ${ }^{25,26}$ Routine MR images are magnitude images (ie, the strength of the MR signal intensity is translated into a grayscale value for display); thus, contrast is mainly related to tissue properties (eg, proton attenuation, $\mathrm{T} 1, \mathrm{~T} 2$, or $\mathrm{T} 22^{*}$ relaxation). Phase information may be obtained at the same time as the magnitude but is typically ignored. Most iron compounds are paramagnetic, meaning generally that they have unpaired electrons and generate weak local fields that add to the strength of the externally applied magnetic field. Diamagnetic substances, such as calcium, usually have no unpaired electrons and subtract from the external field. Regardless of whether the source is paramagnetic or diamagnetic, a change will be produced in the local field, resulting in spatial variations in phase. Phase images, then, measure local changes in susceptibility and can be used either on their own or as the basis for creating a mask to enhance or suppress features of a magnitude image. $3,19,27$

A drawback with phase images is that they often include low-spatial-frequency artifacts from imperfectly shimmed fields or from air or tissue interfaces. With software such as

\begin{tabular}{lc}
\hline Table 1: Subject count per age decade & \\
\hline Decade & No. of Subjects \\
\hline 1st & 19 \\
2nd & 22 \\
3rd & 13 \\
4th & 10 \\
5th & 17 \\
6th & 18 \\
7th & 11 \\
8th & 13 \\
9th & 11 \\
\hline
\end{tabular}

SPIN (signal intensity processing in NMR, developed by the MRI Institute for Biomedical Research, Detroit, Mich), several postprocessing steps are performed in SWI. The phase-contrast image is first high-pass filtered to reduce the unwanted, low-spatial-frequency components, leaving high-frequency field variations created by small anatomic structures virtually intact. A phase mask, which may be "positive" or "negative," is then created. A negative mask involves setting positive phase values (between 0 and $+180^{\circ}$ ) to unity and normalizing the negative phase values (from 0 to $-180^{\circ}$ ) to a gray-scale of values ranging linearly from 1 to 0 . This normalized phase mask is then multiplied against the original magnitude image (typically 3 to 5 times) to yield images that maximize the negative intensities of parenchymal mineralization. ${ }^{20}$ Minimum intensity projection (mIP) images of 2 to 4 sections are then used to display the processed data. The presence of iron in the basal ganglia has been shown to correlate directly with phase and thereby enhance the SWI contrast. ${ }^{3,21,24}$ We have used this property to examine the extent of mineralization seen in the deep gray matter. Because no normative data have yet been published for this state-of-the-art imaging technique, our study intends to establish the course of mineralization by decade in the deep gray matter, as determined by SWI.

\section{Materials and Methods}

\section{Subjects}

In our study, we retrospectively selected 134 patients (68 male, 66 female) who were imaged between June 2001 and November 2004 (Table 1). These patients presented with a variety of health complaints and ranged in age from 1 to 88 years (mean age, 40.2 years; SD, 25.8 years). At least 10 patients were recruited for each decade. Inclusion criteria comprised a normal conventional MR imaging examination (sagittal T1, axial T2, and axial fluid-attenuated inversion recovery [FLAIR] sequences) and acquisition of a normal SWI sequence.

We obtained written informed consent from the patients for the use of data before they underwent MR imaging examinations. The Loma Linda University Medical Center (LLUMC) Institutional Review Board and the University of Saskatchewan Ethics Review Board approved the study.

\section{MR Imaging Procedure}

We performed imaging for this study on a conventional 1.5T wholebody system (Vision; Siemens, Erlangen, Germany), using a standard quadrature head coil. Routine MR imaging included a sagittal T1weighted spin-echo (SE) sequence (TR, 510-700 msec; TE, 12-14 msec; number of acquisitions, 4; number of sections, $5 \mathrm{~mm}$ thick), an axial T2-weighted SE sequence (TR, 2500-7817 msec; TE, 80-120 
msec; number of acquisitions, 1; number of sections, $5 \mathrm{~mm}$ thick), and an axial FLAIR sequence (TR, 9000-9999 msec; TE, 110-119 msec; number of acquisitions, 1 ; number of sections, $5 \mathrm{~mm}$ thick).

We acquired SWI using a 3D FLASH sequence with TR, $57 \mathrm{msec}$; TE, $40 \mathrm{msec}$; flip angle, $20^{\circ}$; FOV, $160 \times 256 \mathrm{~mm}^{2}$; and matrix, $160 \times$ 512. The sequence captured 32 axial sections with a thickness of 2 $\mathrm{mm}$, a low bandwidth $(78 \mathrm{~Hz} /$ pixel), and gradient moment nulling in all 3 orthogonal directions. We postprocessed SWI phase images using a high-pass filter and then converted the images into negative phase masks, which were multiplied 4 times into the corresponding magnitude images. Two-section mIPs were then created from the phase-enhanced magnitude images. Images were reviewed on a DS3000 Impax workstation, version 4.5, (Agfa-Gevaert, Mortsel, Belgium).

\section{Data Collection}

We established a system to describe mineralization in the regions of the putamen, globus pallidus, caudate nucleus, thalamus, red nucleus, and substantia nigra. Because it has been found that lateral differences in the caudate nucleus can exist, regardless of sex and handedness, ${ }^{28}$ all measurements and descriptions of these areas were taken from the right side of the brain.

The signal intensity values of the putamen and globus pallidus were reported as intensity per area so that an apparent increase in mineral concentration would not simply be the result of the decrease in volume that occurs in some brain structures with age. To ensure consistency in measurement and grading, all subjects were reviewed by the same neuroradiologist.

\section{Putamen}

We measured the maximum area and relative signal intensity of the putamen by drawing a region of interest around the single largest view of the putamen (Fig 1). Mineralization was graded according to a modified scale of hypointensity in the putamen, or mSHIP (Table 2), based on a scale developed by Steffens et $\mathrm{al}^{29}$ to describe the posterolateral to anteromedial gradient of mineralization of the putamen with age (Fig 2).

\section{Globus Pallidus}

We measured the maximum area and relative signal intensity of the globus pallidus by drawing a region of interest around the single largest view of the globus pallidus (Fig 1). The presence of mineralization of the medial and lateral aspects of the globus pallidus was assessed (Fig 3). The occurrence of bands of hypointensity within the globus pallidus (Fig 4) was documented and referred to as "waves." We then established a scoring system for the number of waves (Table 3).

\section{Other Regions}

The presence or absence of mineralization was documented in the red nucleus, substantia nigra, caudate head, thalamus, and fascicula nigrale (Fig 5). If mineralization were found to be present in the red nucleus, substantia nigra, or caudate head, we obtained a relative measurement of signal intensity by placing a region of interest over the structure.

\section{Statistical Analysis}

As we determined continuous variables and correlation coefficients, we compared intensity by area for both the putamen and globus pallidus with age. The intensities of the caudate nucleus, substantia nigra, and red nucleus also correlated with age as continuous variables. We

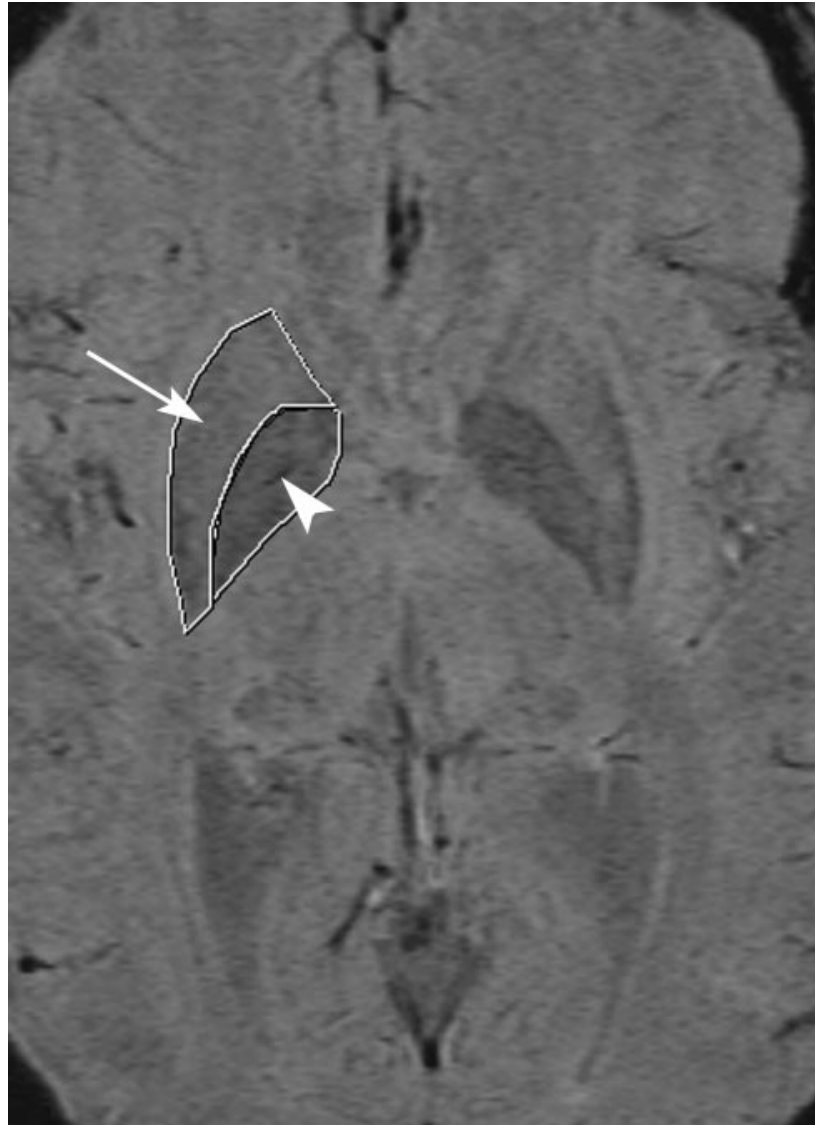

Fig 1. Axial SWI image demonstrating a region of interest drawn around the putamen (arrow) and globus pallidus (arrowhead)

\begin{tabular}{|c|c|}
\hline Grade & Definition \\
\hline 0 & No mineralization \\
\hline 1 & Mineralization of the posterolateral aspect of the putamen \\
\hline 2 & Mineralization of the lateral half of the putamen \\
\hline 3 & $\begin{array}{l}\text { Mineralization of the lateral half and the posteromedial } \\
\text { aspect of the putamen }\end{array}$ \\
\hline 4 & Mineralization of the entire putamen \\
\hline
\end{tabular}

compared both putamen grade and globus pallidus "waves" with age as categoric variables by using $\chi^{2}$ analysis. A $P$ value of $<.05$ was deemed to be statistically significant. Bonferroni correction was completed for multiple comparisons of categoric data.

On the basis of the theory that SWI intensity in our study should be inversely correlated with iron concentration, we attempted to fit an inverse form of the Hallgren and Sourander ${ }^{1}$ equations for iron concentration versus age:

$$
y=\left(y_{0}-A e^{-b x}\right)^{-1}
$$

where $y$ is the intensity, $b$ is a time constant, $x$ is the age in years, $y_{0}-$ $\mathrm{A}$ is the intensity at 0 years, and $A$ affects the rate of change of intensity with age.

\section{Results}

We documented increasing mineralization with age in all deep gray matter areas evaluated (substantia nigra: $r=-0.6, P<$ .01 ; caudate nucleus: $r=-0.6, P<.01$; red nucleus: $r=-0.6$, $P<.01$; and thalamus: $r=-0.6, P<.0001)$. The intensity per 


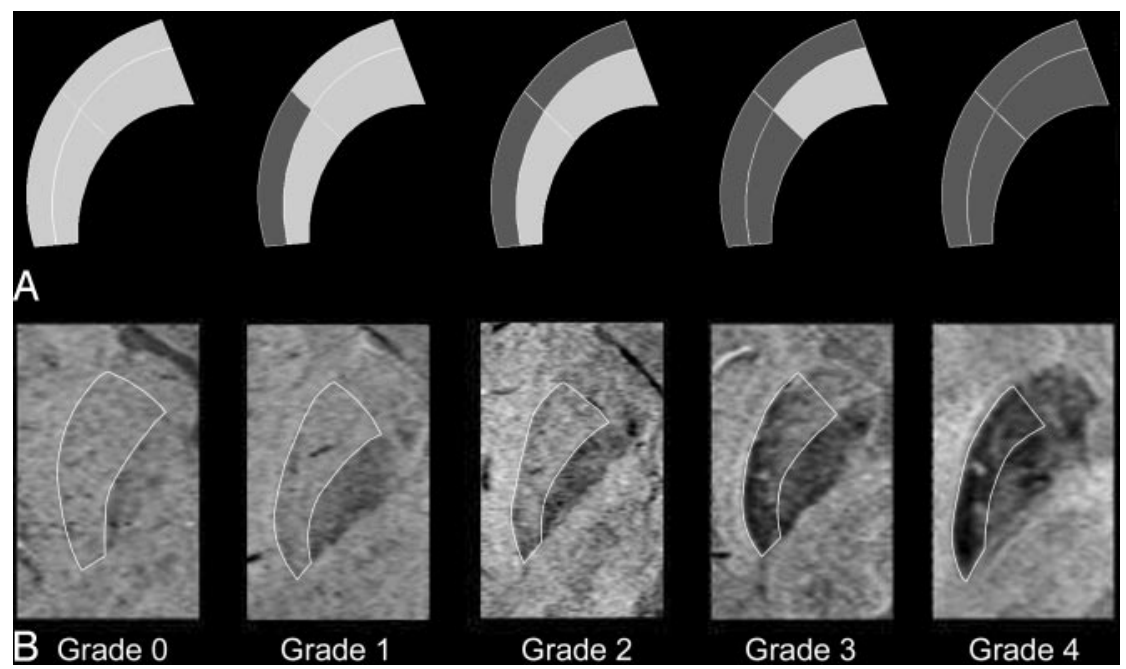

Fig 2. A schematic drawing of the putamen $(A)$ and corresponding axial SWI images $(B)$ demonstrating the mSHIP scale (grades 0 to 4).

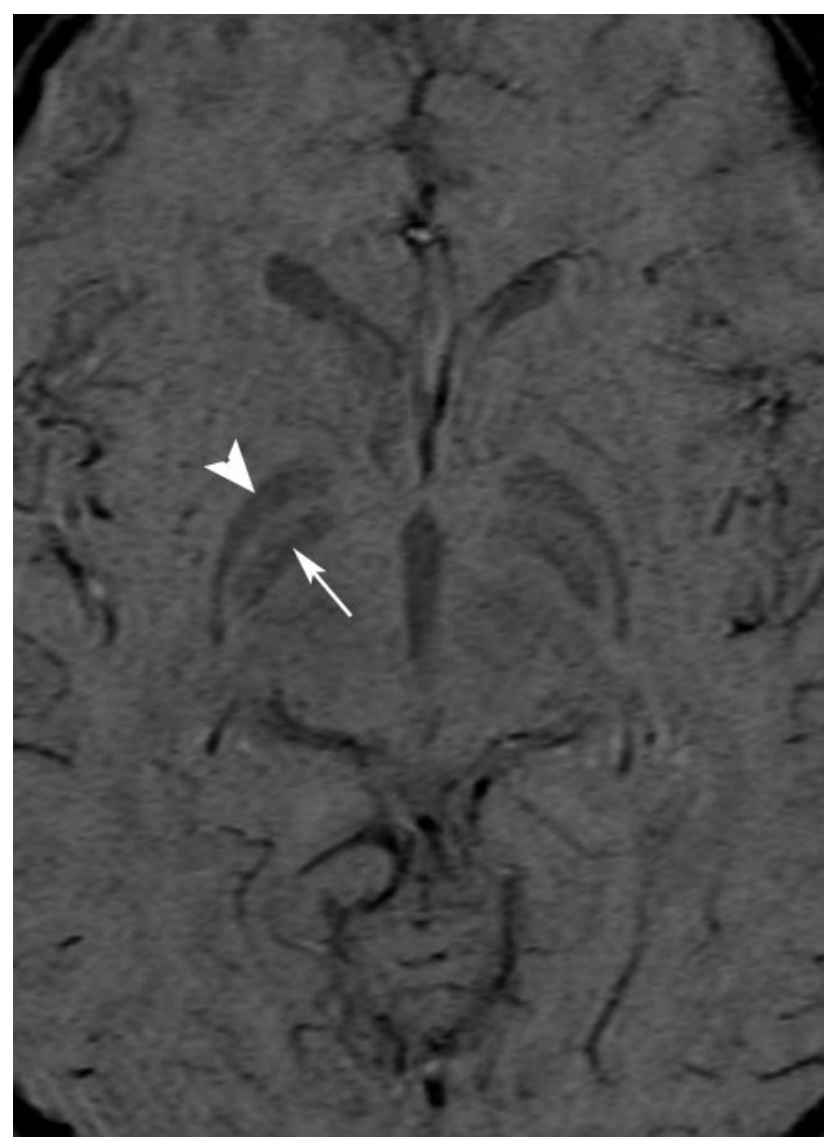

Fig 3. Axial SWI image through the globus pallidus demonstrates mineralization of the medial (arrow) and lateral (arrowhead) aspects of the nucleus.

area of the putamen correlated strongly with age $(r=-0.5, P$ $<$.01) (Fig 6), and the intensity per area of the globus pallidus also decreased with age $(r=-0.6, P<.0001)$ (Fig 7). As shown in Table 4, we obtained good fits of Equation 1 to intensity data for all regions examined, except the caudate nucleus. We found that the intensity of the caudate nucleus exhibits a fairly linear decrease with increasing age and therefore is not well modeled by the inverse exponential decay equation.

A systematic pattern of mineralization related to the putamen was noted, extending from posterolateral to anteromedial with age (grade vs age category, $r=0.6, P<.01$ ) (Fig 8 ).
An increase in the number of "waves" present in the globus pallidus was associated with increased age by category $(r=0.5$, $P<.01$ ) (Fig 9). Subjects younger than 3 years did not exhibit medial and lateral bands of mineralization in the globus pallidus. However, from age 3 until the late 20's, all patients were found to have the characteristic medial-lateral pattern as shown in Fig 3. After the third decade, this pattern becomes less distinct as overall mineralization increases in the globus pallidus. A tract, the fascicula nigrale, was noted extending from the substantia nigra to the medial aspect of the globus pallidus (Fig 5).

\section{Discussion}

It is well documented that iron content in the brain increases with age, particularly in the basal ganglia, and that abnormal levels of iron in the CNS are seen in several neurodegenerative diseases. Understanding the appearance of the aging brain is an important step in the interpretation of imaging in the diseased brain.

Of the metals found in postmortem analysis of mineralization of the basal ganglia, iron, manganese, copper, and calcium may possibly be detected by SWI., ${ }^{3,15,17,21,30}$ Nonpathologic concentrations of manganese and copper are considered too low to be measurable $e^{3,30}$ and thus are not considered in the present analysis. Calcium deposits are typically diamagnetic, resulting in positive phase shifts. ${ }^{7}$ Because our phase mask was designed to enhance the negative phase shifts produced by paramagnetic compounds, hypointense regions in our study were most likely caused by the presence of iron compounds. That is, the intensity of our images tended to correlate inversely with iron concentration.

Because deoxyhemoglobin is also paramagnetic, blood may confound nonheme iron quantification by SWI., 13,21 However, as pointed out by Ogg et al, ${ }^{21}$ there seems to be no correlation between regional hypointensity and blood volume or oxygen use. Moreover, aging tends to decrease cerebral blood volume, which should, in turn, reduce tissue paramagnetism and thus image hypointensity, contrary to findings. Although heme iron may lower the overall intensity of susceptibility-weighed images, it is unlikely to be responsible for regional variations or the observed aging trends.

In general, SWI illustrates a correlation between age and 


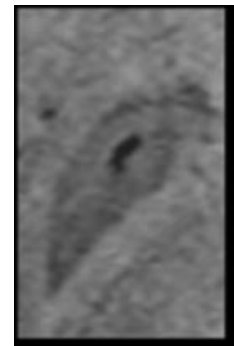

1 Wave

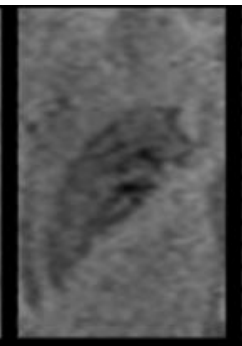

2 Waves

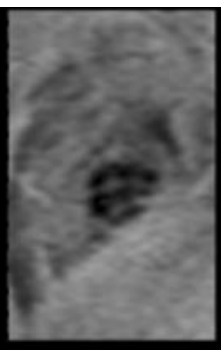

3 Waves

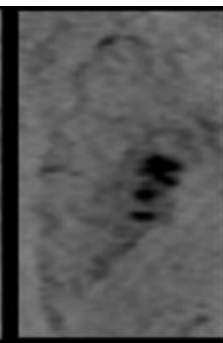

4 Waves

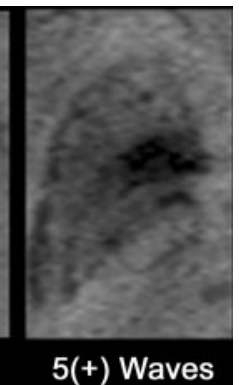

Fig 4. Axial SWI images through the right globus pallidus. Images $A$ to $E$ demonstrate increasing numbers of waves with the final image $(E)$, showing conglomerate waves.

\begin{tabular}{ll}
\hline Table 3: Globus pallidus wave score & \\
\hline Score & \multicolumn{1}{c}{ Definition } \\
\hline 0 & No waves \\
1 & 1 wave \\
2 & 2 waves \\
3 & 3 waves \\
4 & 4 waves \\
5 & 5 waves or confluent waves \\
\hline
\end{tabular}

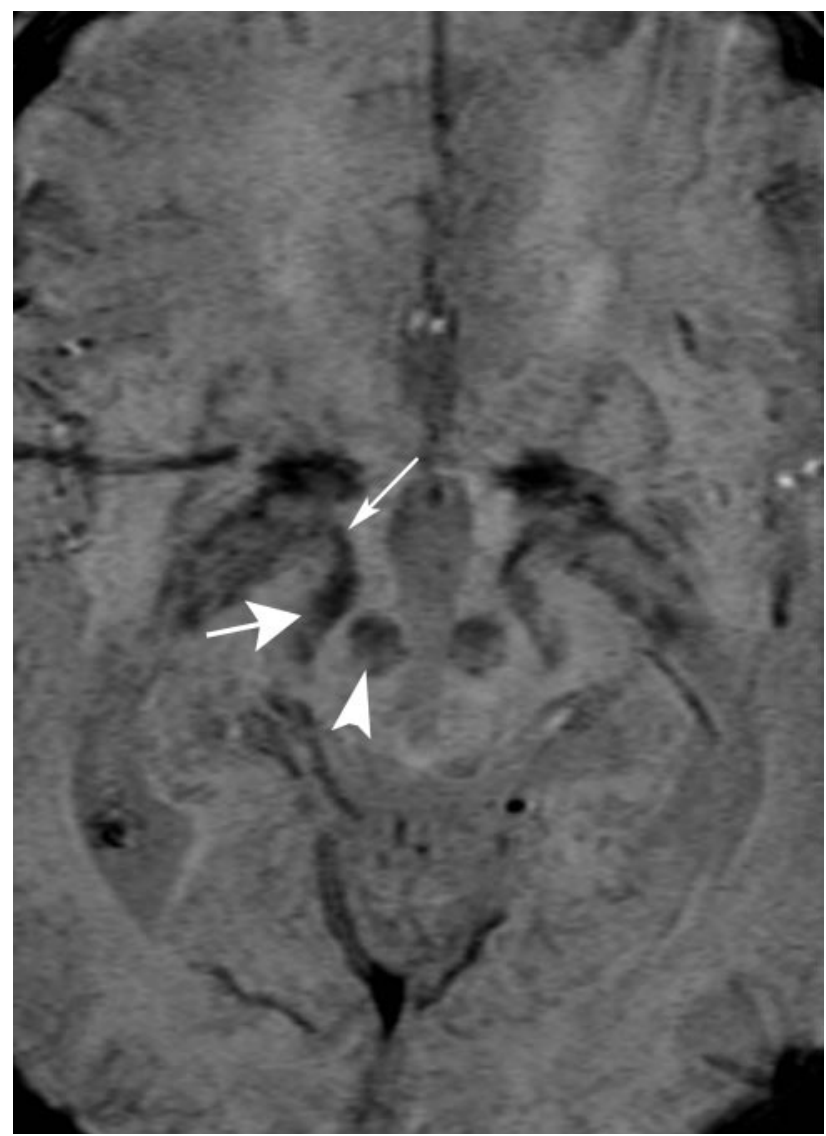

Fig 5. Axial SWI image through the level of the midbrain demonstrating the red nucleus (arrowhead), substantia nigra (thick arrow), and fascicula nigrale (thin arrow).

decreased signal intensity in the basal ganglia. The globus pallidus, putamen, red nucleus, and substantia nigra all demonstrate a steep decrease in intensity during the first 2 decades of life. The volume-corrected intensities for the globus pallidus and putamen essentially plateau by the fifth decade, whereas the red nucleus and substantia nigra continue to have a gradually declining intensity through life. These findings agree quite well with the landmark work of Hallgren and Sourander ${ }^{1}$ as well as with the iron-staining experiments by Drayer et al. ${ }^{17}$
The initial rapid mineralization of the globus pallidus has been reported in other MR studies, ${ }^{14,15,31}$ though there is some disagreement about what occurs later in life. Hallgren and Sourander ${ }^{1}$ and Schenker et $\mathrm{al}^{31}$ postulate that the globus pallidus reaches iron saturation before the age of 40 , whereas Milton et $\mathrm{al}^{14}$ observe that middle-aged and elderly adults show increased iron content compared with young adults. We found that raw globus pallidus intensity does continue to decline gradually through adulthood, but when intensity is corrected for reduced volume, we see a plateau (Fig 7). We note that our putamen data show a steeper decrease in intensity in the first 3 decades (implying greater increase in mineralization) than was determined by Hallgren and Sourander. ${ }^{1}$ However, because their study strictly measured iron concentration, the discrepancy may be the result of the ability of SWI to detect an increase of other minerals in the putamen.

We found that the intensity-versus-age curve for the caudate does not exhibit the same steep decrease as the other structures but rather a more intermediate, steady progression. These observations concur once again with the postmortem studies. ${ }^{1,17}$ Our data also agree with previous work showing that mineralization of the putamen progresses posterolaterally to anteromedially. ${ }^{29}$ In addition, we found a pattern of increasing wave-count within the globus pallidus with age.

Qualitative differences between our findings and those of some previous MR imaging studies may suggest that SWI is more sensitive to lower mineral concentrations than T2 shortening. Contrary to our observations, Ketonen ${ }^{15}$ and Milton et $\mathrm{al}^{14}$ report no putaminal mineralization until the sixth or seventh decade of life and no hypointensity in the caudate at any age. Milton et $\mathrm{al}^{14}$ also report constant mineralization of the red nucleus and substantia nigra at all ages.

The mechanism by which iron is deposited with age is still unknown. In a recent, detailed study of the vascular supply to the striatum, Feekes and Cassell ${ }^{6}$ determined that the striatum could be divided into 3 regions of independent vascular beds: anterior-ventral, remaining medial, and remaining lateral. The medial and lateral portions of the putamen are thus supplied by separate arteries, which may provide a clue as to why putaminal mineralization proceeds in a posterolateral to anteromedial pattern. It has also been postulated that abnormal accumulation of iron may not be related to uptake but to abnormal transport. ${ }^{15}$ Transferrin receptors seem to be densest not at the sites of highest iron concentration, but in regions that send their main fibers to these sites, suggesting that iron may be transported axonally. ${ }^{10,17}$ An interesting tract, the fascicula nigrale, was identified adjacent to the substantia nigra in some of the 
Putamen: Corrected Intensity vs. Age

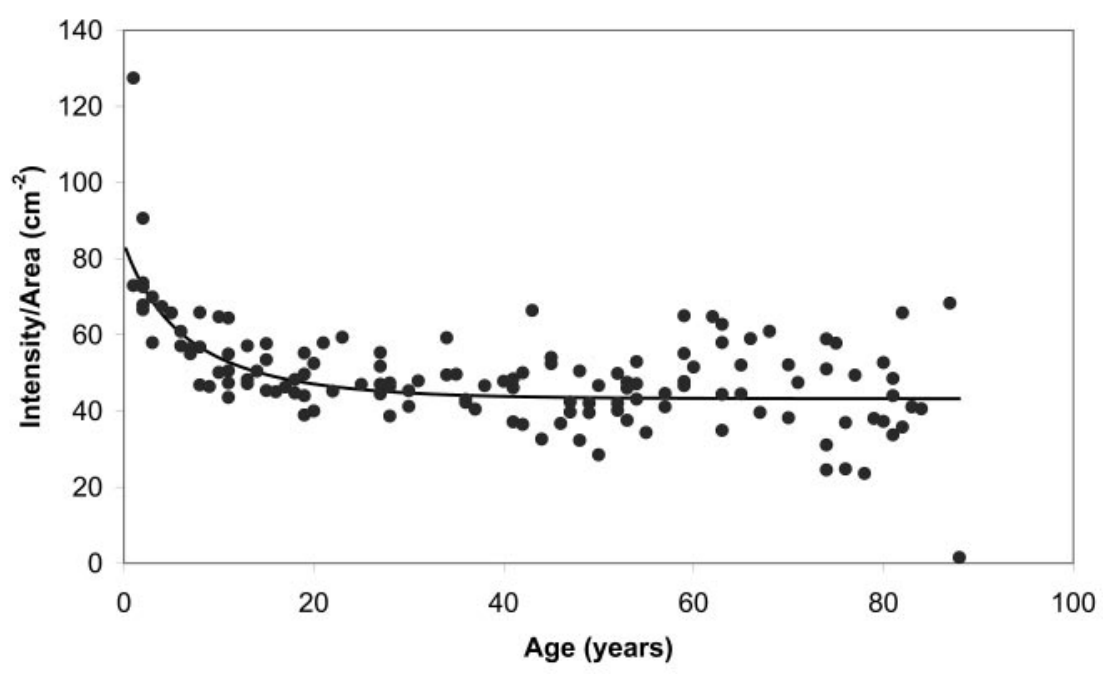

Globus Pallidus: Corrected Intensity vs. Age

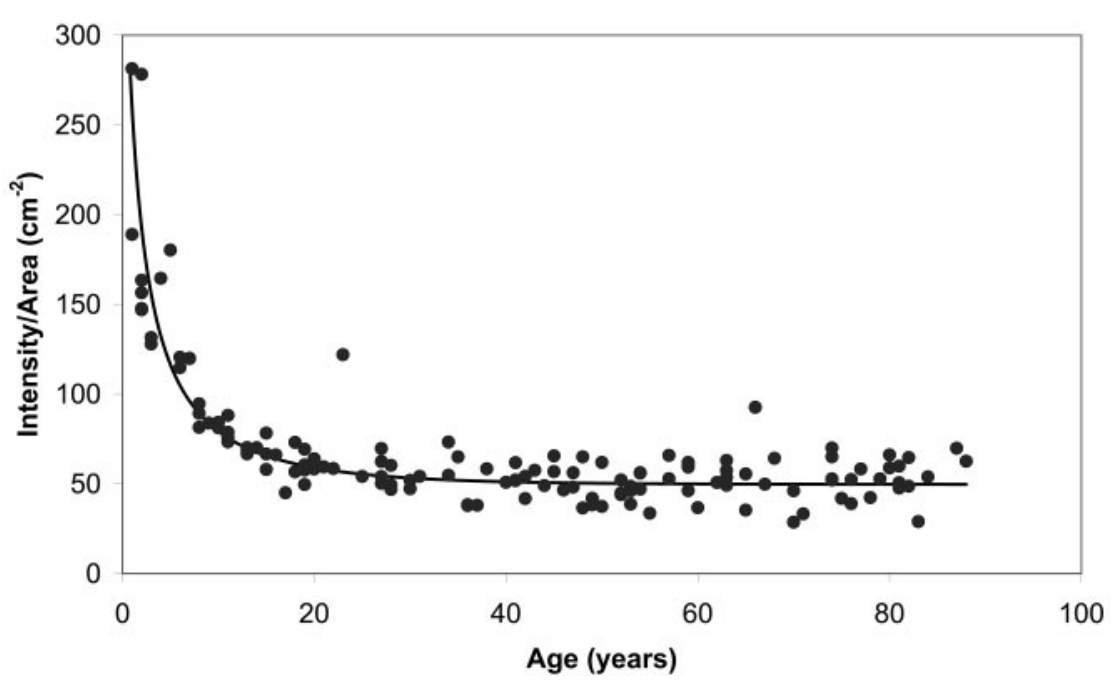

Fig 6. Putaminal intensity per area decreases with age. The line represents Equation 1, with the parameter for the putamen as given in Table 4 .
Fig 7. Intensity per area of the globus pallidus decreases with age. The line represents Equation 1, with the parameter for the globus pallidus as given in Table 4 .
Table 4: Fit of intensity vs age data to inverse iron concentration curves*

\begin{tabular}{lcccc}
\hline & & & \multicolumn{2}{c}{ Correlation, } \\
& $y_{0}$ & $A$ & $b$ & $r$ \\
\hline Globus pallidus & 0.020 & 0.018 & 0.09 & 0.8 \\
Putamen & 0.023 & 0.011 & 0.09 & 0.5 \\
Substantia nigra & 0.012 & 0.0065 & 0.04 & 0.5 \\
\hline
\end{tabular}

* See Equation 1.

subjects. This finding may play a role in dynamic iron transport in the extrapyramidal system.

One shortcoming of our study was the lack of detailed medical histories for the subjects. Confounding factors influencing findings on SWI may have been present, despite all subjects having normal conventional MR imaging. The standard deviation of signal intensity increased with age, raising the possibility that ageassociated pathologic changes may be occurring in some of these patients. Another potential drawback arose from having only analyzed structures in the right hemisphere. Some researchers have found hemispheric differences in mineralization with certain diseases. For example, Steffens et $\mathrm{al}^{32}$ found that older patients who suffered from depression exhibited greater iron concentration in the left putamen. Recently, Bartzokis et $\mathrm{al}^{33}$ have suggested that normal mineralization for some areas of the brain may vary with sex. On the basis of past work that found no significant variation, our study did not differentiate between the sexes.

We are currently working on a comparative study using a synchrotron imaging technique called $\mathrm{x}$-ray absorption spectroscopy (XAS), which is capable of generating precise maps of metals in the brain. ${ }^{34}$ Because SWI lacks chemical sensitivity, the interpretation of SWI image intensity may be improved through comparison with the XAS maps of metals. In addition, the high resolution achievable with XAS imaging may uncover new information about the co-localization and deposition of different metals, and about pathways of metal storage in the brain. Such knowledge may allow us to enhance the appearance of particular metals on SWI by refining the phase-masking step.

\section{Conclusion}

This paper documents the course and pattern of mineralization in the deep gray matter with age with the use of SWI. 


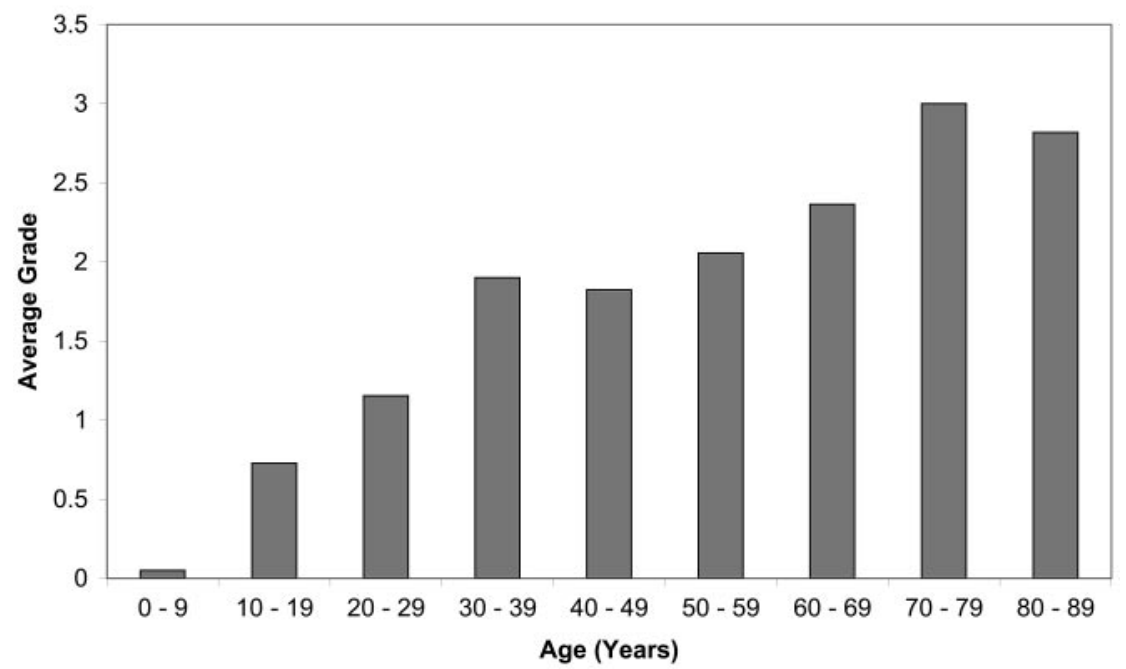

Globus Pallidus: Average Wave Count vs. Age

Fig 9. Globus pallidus "waves" increase with age.

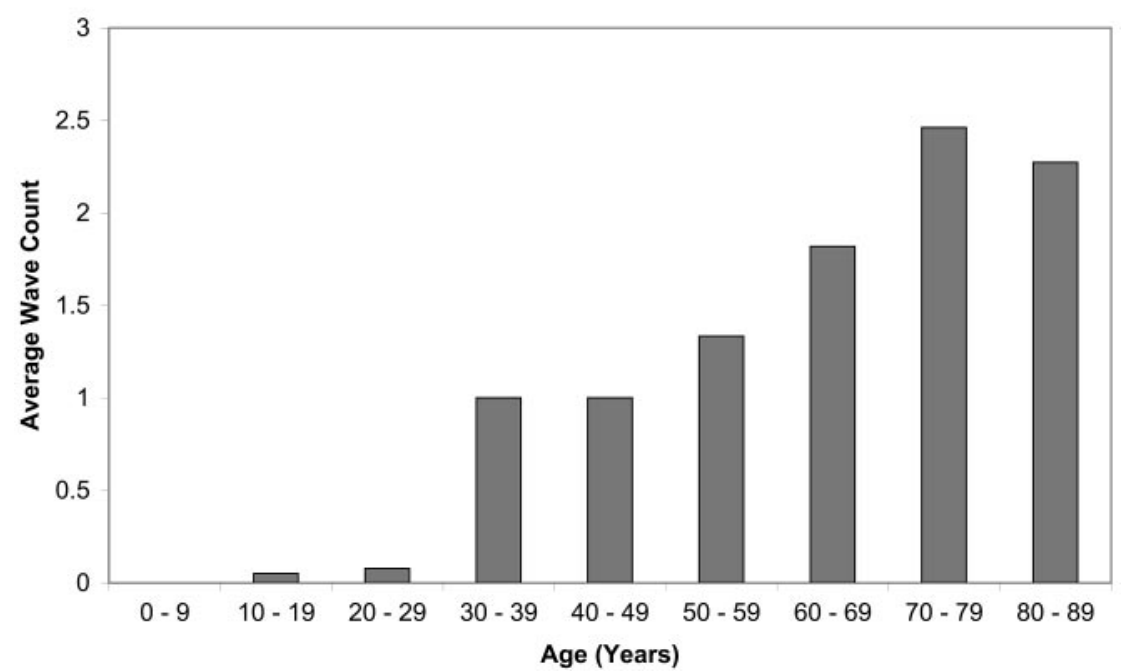

These findings may play a role in the evaluation of diseased brains in the future, though it must be reiterated that despite normal conventional imaging, the subjects in our study encompassed a heterogeneous group. We will soon carry out an additional study comparing synchrotron-based XAS imaging with SWI. This study will further quantize and characterize minerals in the basal ganglia of both control and diseased brains.

\section{References}

1. Hallgren B, Sourander P. The effect of age on the non-haemin iron in the human brain. J Neurochem 1958;3:41-51

2. Dobson J. Nanoscale biogenic iron oxides and neurodegenerative disease. FEBS Lett 2001;496:1-5

3. Haacke EM, Cheng NY, House MJ, et al. Imaging iron stores in the brain using magnetic resonance imaging. Magn Reson Imaging 2005;23:1-25

4. Koeppen AH. The history of iron in the brain. I Neurol Sci 1995;134 Suppl:1-9

5. Casanova MF, Araque JM. Mineralization of the basal ganglia: implications for neuropsychiatry, pathology and neuroimaging. Psychiatry Res 2003;121:59-87

6. Feekes JA, Cassell MD. The vascular supply of the functional compartments of the human striatum. Brain 2006;129:2189-201

7. Yamada N, Imakita S, Sakuma T, et al. Intracranial calcification on gradientecho phase image: depiction of diamagnetic susceptibility. Radiology 1996;198:171-78
8. Cohen CR, Duchesneau PM, Weinstein MA. Calcification of the basal ganglia as visualized by computed tomography. Radiology 1980;134:97-99

9. Vermersch P, Leys D, Pruvo JP, et al. Parkinson's disease and basal ganglia calcifications: prevalence and clinico-radiological correlations. Clin Neurol Neurosurg 1992;94:213-17

10. Aoki S, Okada Y, Nishimura K, et al. Normal deposition of brain iron in childhood and adolescence: MR imaging at 1.5 T. Radiology 1989;172:381-85

11. Bartzokis G, Mintz J, Sultzer D, et al. In vivo MR evaluation of age-related increases in brain iron. AJNR Am J Neuroradiol 1994;15:1129-38

12. Drayer BP. Imaging of the aging brain. Part II. Pathologic conditions. Radiology 1988;166:797-806

13. Gelman N, Gorell JM, Barker PB, et al. MR imaging of human brain at $3.0 \mathrm{~T}$ preliminary report on transverse relaxation rates and relation to estimated iron content. Radiology 1999;210:759-67

14. Milton WJ, Atlas SW, Lexa FJ, et al. Deep gray matter hypointensity patterns with aging in healthy adults: MR imaging at 1.5 T. Radiology 1991; 181:715-19

15. Ketonen LM. Neuroimaging of the aging brain. Neurol Clin 1998;16:581-98

16. Chen JC, Hardy PA, Kucharczyk W, et al. MR of human postmortem brain tissue: correlative study between T2 and assays of iron and ferritin in Parkinson and Huntington disease. AJNR Am J Neuroradiol 1993;14:275-81

17. Drayer B, Burger P, Darwin R, et al. MRI of brain iron. AJR Am J Roentgenol 1986;147:103-10

18. Ma J, Wehrli FW. Method for image-based measurement of the reversible and irreversible contribution to the transverse-relaxation rate. J Magn Reson B 1996;111:61-69

19. Reichenbach JR, Venkatesan R, Schillinger DJ, et al. Small vessels in the human 
brain: MR venography with deoxyhemoglobin as an intrinsic contrast agent. Radiology 1997;204:272-77

20. Haacke EM, Xu Y, Cheng YC, et al. Susceptibility weighted imaging (SWI). Magn Reson Med 2004;52:612-18

21. Ogg RJ, Langston JW, Haacke EM, et al. The correlation between phase shifts in gradient-echo MR images and regional brain iron concentration. Magn Reson Imaging 1999;17:1141-48

22. Cho ZH, Ro YM, Lim TH. NMR venography using the susceptibility effect produced by deoxyhemoglobin. Magn Reson Med 1992;28:25-38

23. Lee BC, Vo KD, Kido DK, et al. MR high-resolution blood oxygenation leveldependent venography of occult (low-flow) vascular lesions. AJNR Am J Neuroradiol 1999;20:1239-42

24. Sehgal V, Delproposto Z, Haacke EM, et al. Clinical applications of neuroimaging with susceptibility-weighted imaging. J Magn Reson Imaging 2005;22:439-50

25. Tong KA, Ashwal S, Holshouser BA, et al. Hemorrhagic shearing lesions in children and adolescents with posttraumatic diffuse axonal injury: improved detection and initial results. Radiology 2003;227:332-39

26. Tong KA, Ashwal S, Holshouser BA, et al. Diffuse axonal injury in children: clinical correlation with hemorrhagic lesions. Ann Neurol 2004;56:36-50
27. Rauscher A, Sedlacik J, Barth M, et al. Magnetic susceptibility-weighted MR phase imaging of the human brain. AJNR Am J Neuroradiol 2005;26:736-42

28. Ifthikharuddin SF, Shrier DA, Numaguchi Y, et al. MR volumetric analysis of the human basal ganglia: normative data. Acad Radiol 2000;7:627-34

29. Steffens DC, McDonald WM, Tupler LA, et al. Magnetic resonance imaging changes in putamen nuclei iron content and distribution in normal subjects. Psychiatry Res 1996;68:55-61

30. Schenck JF. Magnetic resonance imaging of brain iron. J Neurol Sci 2003;207:99-102

31. Schenker C, Meier D, Wichmann W, et al. Age distribution and iron dependency of the $\mathrm{T} 2$ relaxation time in the globus pallidus and putamen. Neuroradiology 1993;35:119-24

32. Steffens DC, Tupler LA, Ranga K, et al. Magnetic resonance imaging signal hypointensity and iron content of putamen nuclei in elderly depressed patients. Psychiatry Res 1998;83:95-103

33. Bartzokis G, Tishler TA, Lu PH, et al. Brain ferritin iron may influence age- and gender-related risks of neurodegeneration. Neurobiol Aging 2007;28:414-23

34. Collingwood JF, Mikhaylova A, Davidson M, et al. In situ characterization and mapping of iron compounds in Alzheimer's disease tissue. J Alzheimers Dis $2005 ; 7: 267-72$ 